\title{
Bethe subalgebras in Braided Yangians and Gaudin-type models
}

\author{
Dimitri Gurevich* \\ Université de Valenciennes, EA 4015-LAMAV \\ F-59313 Valenciennes, France \\ and \\ Interdisciplinary Scientific Center Poncelet (ISCP, UMI 2615 du CNRS) \\ Moscow 119002, Russian Federation \\ Pavel Saponov ${ }^{\dagger}$ \\ National Research University Higher School of Economics, \\ 20 Myasnitskaya Ulitsa, Moscow 101000, Russian Federation \\ and \\ Institute for High Energy Physics, NRC "Kurchatov Institute" \\ Protvino 142281, Russian Federation \\ Alexei Slinkin \\ National Research University Higher School of Economics, \\ 20 Myasnitskaya Ulitsa, Moscow 101000, Russian Federation
}

October 9, 2018

\begin{abstract}
In GS1 the notion of braided Yangians of Reflection Equation type was introduced. Each of these algebras is associated with an involutive or Hecke symmetry $R$. Besides, the quantum analogs of certain symmetric polynomials (elementary symmetric ones, power sums) were suggested. In the present paper we show that these quantum symmetric polynomials commute with each other and consequently generate a commutative Bethe subalgebra. As an application, we get some Gaudin-type models and the corresponding Bethe subalgebras.
\end{abstract}

AMS Mathematics Subject Classification, 2010: 81R50, 82B23

Keywords: current braiding, braided Yangian, quantum elementary symmetric polynomials, quantum power sums, quantum Newton identity.

\section{Introduction}

Let $V$ be a finite dimensional vector space over the ground field $\mathbb{C}, \operatorname{dim}_{\mathbb{C}} V=N$. Consider a linear operator $R \in \operatorname{End}\left(V^{\otimes 2}\right)$ which is a solution of the so-called braid relation

$$
R_{1} R_{2} R_{1}=R_{2} R_{1} R_{2} .
$$

Both sides of this braid relation belong to $\operatorname{End}\left(V^{\otimes 3}\right)$. Also, we use the standard shorthand notation $R_{k}=R_{k k+1}$ for the operator $R$ acting in the positions $k$ and $k+1$ of the tensor product $V^{p}, p \geq k+1$.

*gurevich@ihes.fr

†Pavel.Saponov@ihep.ru 
An operator subject to (1.1) will be called a braiding.

In addition, we assume all braidings $R$, we are dealing with, to obey either the Hecke condition

$$
(R-q I)\left(R+q^{-1} I\right)=0, \quad q \in \mathbb{C} \backslash\{0, \pm 1\}
$$

or the condition

$$
R^{2}=I \text {. }
$$

Hereafter, $I$ is the identity operator or matrix. Respectively, we will call such an operator $R$ a Hecke symmetry or an involutive symmetry.

Given a Hecke or involutive symmetry $R$, we can construct some current (i.e. depending on parameters) operators

$$
R(u, v)=R-\frac{\left(q-q^{-1}\right) u I}{u-v},
$$

provided $R$ is a Hecke symmetry, and

$$
R(u, v)=R-\frac{I}{u-v}
$$

provided $R$ is involutive. These current operators satisfy the Quantum Yang-Baxter equation with parameters

$$
R_{1}(u, v) R_{2}(u, w) R_{1}(v, w)=R_{2}(v, w) R_{1}(u, w) R_{2}(u, v) .
$$

The correspondence $R \mapsto R(u, v)$ is called the Baxterization procedure and the resulting operator $R(u, v)$ is called a trigonometric (resp., rational) $R$-matrix.

Since, in fact, the current operator $R(u, v)$ depends on the ratio of parameters $x=u / v$ in the trigonometric case or on their difference $x=u-v$ in the rational case, we also use the notation $R(x)$ for both cases. So, we respectively have

$$
R(x)=R-\frac{\left(q-q^{-1}\right) x}{x-1} I, \quad R(x)=R-\frac{1}{x} I .
$$

We hope this does not lead to a misunderstanding.

In GS1, GS3] the so-called braided Yangian was introduced. It is generated by an $N \times N$ current matrix $L(u)$, which is subject to the system

$$
R_{1}(u, v) L_{\overline{1}}(u) L_{\overline{2}}(v)-L_{\overline{1}}(v) L_{\overline{2}}(u) R_{1}(u, v)=0 .
$$

Hereafter, we use the following notation

$$
L_{\overline{1}}(u)=L_{1}(u), \quad L_{\overline{k+1}}(u)=R_{k} L_{\bar{k}}(u) R_{k}^{-1}, \quad k \geq 1 .
$$

The generating matrix $L(u)$ is assumed to be a formal series in $u$

$$
L(u)=I+\sum_{k=1}^{\infty} L[k] u^{-k},
$$

where the matrices $L[k], k \geq 1$, are called the Laurent coefficients. Consequently, the condition (1.4) leads to an infinite system of relations on the Laurent coefficients $L[k]$. We use the notation $\mathbf{Y}(R)$ for the braided Yangian defined by the system (1.4). Below, we mainly deal with the whole matrix $L(u)$ without using its Laurent coefficients $L[k]$.

Note, that if we set $R=P$ (hereafter, $P$ stands for the usual flip) the system (1.4) gives rise to the Drinfeld's Yangians $\mathbf{Y}(g l(N))$. By the initial definition, $\mathbf{Y}(g l(N))$ is an algebra, generated by entries of the corresponding Laurent coefficients $L[k]$, whereas entries of the matrix $L(u)$ are treated to be elements of $\mathbf{Y}(g l(N))\left[\left[u^{-1}\right]\right]$. 
Remark 1 A Yangian-like algebra can be defined in a more general way which is based on a compatible pair of braidings $(R, F)$. Recall that according to [IOP] an ordered pair of braidings $(R, F)$ is called compatible if they are subject to the relations

$$
R_{1} F_{2} F_{1}=F_{2} F_{1} R_{2}, \quad R_{2} F_{1} F_{2}=F_{1} F_{2} R_{1} .
$$

Given such a pair, we define the generalized braided Yangian by the relation (1.4) where the copies $L_{\bar{k}}(u)$ are defined with the use of the braiding $F$ :

$$
L_{\overline{1}}(u)=L_{1}(u), \quad L_{\overline{k+1}}(u)=F_{k} L_{\bar{k}}(u) F_{k}^{-1}, \quad k \geq 1 .
$$

For any compatible braidings $R$ and $F$ the use of (1.7) allows us to shift the relations (1.4) to higher positions:

$$
R_{k}(u, v) L_{\bar{k}}(u) L_{\overline{k+1}}(v)=L_{\bar{k}}(v) L_{\overline{k+1}}(u) R_{k}(u, v), \quad \forall k \geq 1 .
$$

Namely this property is the main "raison d'être" for the notion of compatible braidings.

It is easy to see that if $F=P$ or $F=R$, we get a compatible pair $(R, F)$ for any braiding $R$. In order to distinguish these specific cases we call the algebra $\mathbf{Y}(R)$ the braided Yangian of Reflection Equation (RE) type, while for $F=P$ we call the corresponding algebra the Yangian of RTT type. If $R$ is the Hecke symmetry coming from the quantum group $U_{q}(s l(N))$, the corresponding Yangian of RTT type is somewhere called the $q$-Yangian (see [M]).

Note, that analogs of certain symmetric polynomials and the corresponding Bethe subalgebras as well as some analogs of the Newton and Cayley-Hamilton identities can be constructed in all braided Yangians (not only in these of RE type). However, the braided Yangians of RE type have a lot of specific properties, which distinguish them from other braided Yangians. Thus, as was shown in [GS1, the evaluation morphisms in the braided Yangians of RE type are more similar to these in the Drinfeld's Yangians $\mathbf{Y}(g l(N))$. Moreover, the quantum determinant (the higher order elementary symmetric polynomial) is always central in any braided Yangian of RE type, while for other types of braided Yangians it is not true in general (see GS1]).

In the present paper we show that if $R$ is an even symmetry, then the elementary symmetric polynomials in the corresponding braided Yangian of RE type commute with each other and consequently they generate a commutative subalgebra called the Bethe one. Also, we define some Gaudin-type models and by applying the Talalaev's method we construct their Bethe subalgebras, provided $R$ is an involutive symmetry. We call these models braided Gaudin ones.

The paper is organized as follows. The Bethe subalgebras in the braided Yangians are constructed in the next section. Their commutativity is established in section 3 . In section 4 we introduce braided versions of the rational Gaudin model and exhibit their Bethe subalgebras.

Acknowledgement The work of P.S. has been funded by the Russian Academic Excellence Project '5-100' and was also partially supported by the RFBR grant 16-01-00562.

\section{Bethe subalgebras via quantum symmetric polynomials}

We begin this section with the following observation. The current $R$-matrices, we are dealing with, are non-singular except for some isolated values of parameters. Namely, in the rational and trigonometric cases we respectively have

$$
\begin{gathered}
R^{-1}(x)=\frac{x^{2}}{x^{2}-1} R(-x), \quad x \neq \pm 1 \\
R^{-1}(x)=\frac{(x-1)^{2}}{(x-1)^{2}-\lambda^{2} x} R\left(-x^{-1}\right), \quad x \neq q^{ \pm 2}, \quad \lambda \equiv q-q^{-1} .
\end{gathered}
$$


Let us define a family of skew-symmetrizers $A_{1 \ldots k}^{(k)}(R) \in \operatorname{End}\left(V^{\otimes n}\right), n \geq k$, associated with a Hecke symmetriy $R$. They are defined by the following recurrent relations:

$$
A^{(1)}=I, \quad A_{1 \ldots k+1}^{(k+1)}=\frac{k_{q}}{(k+1)_{q}} A_{1 \ldots k}^{(k)}\left(\frac{q^{k}}{k_{q}} I-R_{k}\right) A_{1 \ldots k}^{(k)}, \quad k \geq 1,
$$

where $I$ is the identity matrix of an appropriate size and $k_{q}=\left(q^{k}-q^{-k}\right) /\left(q-q^{-1}\right)$ is the standard notation for $q$-numbers. The parameter $q$ is assumed to be generic: $q^{k} \neq 1, \forall k \in \mathbb{Z}_{+}$, so all $q$-integers are nonzero. The low indeces indicate the positions where these operators are applied.

For an involutive symmetry $R$ the skew-symmetrizers are defined by the same formula (2.1) where we should set $q=1$ and use $k$ instead of $k_{q}$.

Hereafter, we confine ourselves to even skew-invertible symmetries. The bi-rank of these symmetries (Hecke or involutive) are always assumed to be $(m \mid 0) \sqrt{1}$. A definition of even symmetries, bi-rank, and a review of properties of the corresponding symmetries can be found, for example, in [GPS, GS1]. Here, we exhibit some of them:

- For any braiding under consideration there exists an $N \times N$ matrix $C$ with the following properties:

$$
\operatorname{Tr}_{(2)} R_{12} C_{2}=I_{1}, \quad R_{k} C_{k} C_{k+1}=C_{k} C_{k+1} R_{k} \quad \forall k \geq 1, \quad \operatorname{Tr} C=\frac{m_{q}}{q^{m}} .
$$

- With the matrix $C$ we define the $R$-trace of a matrix $X$ (of an appropriate size) with entries from an algebra $\mathcal{A}$ :

$$
\operatorname{Tr}_{R(12 \ldots k)} X=\operatorname{Tr}_{(12 \ldots k)}\left(C_{1} C_{2} \ldots C_{k} X\right), \quad X \in\left(\operatorname{Mat}_{N}(\mathcal{A})\right)^{\otimes k}
$$

The second relation in (2.2) allows us to prove an important cyclic property of the $R$-trace:

$$
\operatorname{Tr}_{R(12 \ldots k)}\left(f\left(R_{1}, R_{2} \ldots R_{k-1}\right) X\right)=\operatorname{Tr}_{R(12 \ldots k)}\left(X f\left(R_{1}, R_{2} \ldots R_{k-1}\right)\right)
$$

for any polynomial $f$ in $R$-matrices and for a matrix $X$ as above.

- The following property of the $R$-trace is of the great importance in what follows:

$$
\operatorname{Tr}_{R(k+1)}\left(R_{k}^{ \pm 1} X_{1 \ldots k} R_{k}^{\mp 1}\right)=I_{1 \ldots k} \operatorname{Tr}_{R(k)}\left(X_{1 \ldots k}\right),
$$

where $X_{1 \ldots k}$ is an operator (a matrix) in the space $V^{\otimes k}$ and $I_{1 \ldots k}$ is the identity operator (matrix) in the same space.

- The skew-symmetrizers (2.1) are idempotents

$$
A^{(k)} A^{(k)}=A^{(k)} \quad \forall k \geq 1,
$$

and they vanish for $k \geq m+1$ :

$$
A^{(m+1)} \equiv 0, \quad \operatorname{rank} A^{(m)}=1 .
$$

Now, we introduce quantum analogs of the elementary symmetric polynomials defined via generating matrix $L(u)$ of a braided Yangian of RE type $\mathbf{Y}(R)$ in the trigonometrical case.

\footnotetext{
${ }^{1}$ Observe that in general $m \neq N$. However, if $R$ equals $P$ or is its deformation, then $m=N$.
} 
Definition 2 The elements

$$
e_{k}(u)=\operatorname{Tr}_{R(12 \ldots k)} A_{12 \ldots k}^{(k)} L_{\overline{1}}(u) L_{\overline{2}}\left(q^{-2} u\right) \ldots L_{\bar{k}}\left(q^{-2(k-1)} u\right), \quad k \geq 1,
$$

are called (quantum) elementary symmetric polynomials in the Yangian of RE type $\mathbf{Y}(R)$.

The subalgebra generated in $\mathbf{Y}(R)$ by the elementary symmetric polynomials is called the Bethe subalgebra.

It should be emphasized that this definition (as well as the definition below of the power sums) is valid without assuming $R$ to be even. However, if $R$ is even of bi-rank $(m \mid 0)$, then there exists the highest elementary symmetric polynomial $e_{m}(u)$ which is called the quantum determinant. Observe that it is always central in the corresponding braided Yangian $\mathbf{Y}(R)$.

In the next section we show that the elementary symmetric polynomials commute with each other and consequently, the Bethe subalgebra is commutative. To this end we need the following lemma.

Lemma 3 The following matrix identity holds true:

$$
\operatorname{Tr}_{R(k+1 \ldots k+p)} A_{k+1 \ldots k+p}^{(p)} L_{\overline{k+1}}(u) L_{\overline{k+2}}\left(q^{-2} u\right) \ldots L_{\overline{k+p}}\left(q^{-2(p-1)} u\right)=I_{12 \ldots k} e_{p}(u),
$$

where $e_{p}(u)$ is an elementary symmetric polynomial defined in 2.7.).

Proof. First of all, note that due to the definition of $L_{\bar{k}}(u)$ and the braid relation for $R$ we have

$$
R_{i}^{ \pm 1} L_{\bar{k}}(u)=L_{\bar{k}}(u) R_{i}^{ \pm 1} \quad \forall i \notin\{k-1, k\} .
$$

Taking into account this property we can write (we omit the arguments in the matrices)

$$
L_{\overline{k+1}} L_{\overline{k+2}}=R_{k} L_{\bar{k}} R_{k}^{-1} R_{k+1} R_{k} L_{\bar{k}} R_{k}^{-1} R_{k+1}^{-1}=R_{k} R_{k+1}\left(L_{\bar{k}} L_{\overline{k+1}}\right) R_{k+1}^{-1} R_{k}^{-1},
$$

where we used the braid relation $R_{k}^{-1} R_{k+1} R_{k}=R_{k+1} R_{k} R_{k+1}^{-1}$ in the middle of the above expression.

Then we continue as follows

$$
L_{\overline{k+1}} L_{\overline{k+2}} L_{\overline{k+3}}=R_{k} R_{k+1} L_{\bar{k}} L_{\overline{k+1}} L_{\overline{k+3}} R_{k+1}^{-1} R_{k}^{-1}=R_{k} R_{k+1} R_{k+2}\left(L_{\bar{k}} L_{\overline{k+1}} L_{\overline{k+2}}\right) R_{k+2}^{-1} R_{k+1}^{-1} R_{k}^{-1}
$$

and so on till the following intermediate result:

$$
L_{\overline{k+1}} \ldots L_{\overline{k+p}}=R_{k} \ldots R_{k+p-1}\left(L_{\bar{k}} \ldots L_{\overline{k+p-1}}\right) R_{k+p-1}^{-1} \ldots R_{k}^{-1} .
$$

Now, with the use of definition (2.1) and the braid relation for $R$ we can show that

$$
A_{k+1 \ldots k+p}^{(p)} R_{k} R_{k+1} \ldots R_{k+p-1}=R_{k} R_{k+1} \ldots R_{k+p-1} A_{k \ldots k+p-1}^{(p)} .
$$

So, we have

$$
\begin{aligned}
\operatorname{Tr}_{R(k+1 \ldots k+p)} & A_{k+1 \ldots k+p}^{(p)} L_{\overline{k+1}} L_{\overline{k+2}} \ldots L_{\overline{k+p}}= \\
& \operatorname{Tr}_{R(k+1 \ldots k+p)} R_{k} R_{k+1} \ldots R_{k+p-1} X_{1 \ldots k+p-1} R_{k+p-1}^{-1} \ldots R_{k}^{-1}
\end{aligned}
$$

where for the sake of simplicity we denoted $X_{1 \ldots k+p-1}=A_{k \ldots k+p-1}^{(p)} L_{\bar{k}} \ldots L_{\overline{k+p-1}}$.

At last, applying the relation (2.5) $p$ times we get

$$
\operatorname{Tr}_{R(k+1 \ldots k+p)} A_{k+1 \ldots k+p}^{(p)} L_{\overline{k+1}} L_{\overline{k+2}} \ldots L_{\overline{k+p}}=I_{1 \ldots k} \operatorname{Tr}_{R(k \ldots k+p-1)} A_{k \ldots k+p-1}^{(p)} L_{\bar{k}} L_{\overline{k+1}} \ldots L_{\overline{k+p-1}} .
$$

Thus, we have shifted down by one the numbers of spaces where the $R$-trace is taken. The rest of the proof is evident: applying the same steps as above, we finally come to the relation (2.8) 
Remark 4 Note that the relation (2.8) is also valid for a generalized Yangian associated with a compatible pair $(R, F)$, where $R$ is assumed to be a Hecke symmetry. The proof is similar to that above except for the definition of trace: the $R$-trace should be changed for the $F$-trace. This means that the matrix $C$ in definition (2.3) corresponds to the braiding $F$ instead of $R$.

It is natural to ask whether there exist analogs of other symmetric polynomials in the algebra $\mathbf{Y}(R)$ ? We are able to give the positive answer for the so-called power sums and full symmetric polynomials. As for the quantum analogs of the Schur polynomials, we do not know their consistent definition.

Definition 5 The elements

$$
p_{k}(u)=\operatorname{Tr}_{R(1 \ldots k)}\left(L_{\overline{1}}\left(q^{-2(k-1)} u\right) L_{\overline{2}}\left(q^{-2(k-2)} u\right) \ldots L_{\bar{k}}(u) R_{k-1} \ldots R_{2} R_{1}\right), \quad k \geq 1 .
$$

are called the quantum power sums.

The sets of elementary symmetric polynomials and power sums are related by a series of quantum analogs of the Newton identities [GS1]:

$$
k_{q} e_{k}(u)-q^{k-1} p_{1}\left(q^{-2(k-1)} u\right) e_{k-1}(u)+q^{k-2} p_{2}\left(q^{-2(k-2)} u\right) e_{k-2}(u)+\cdots+(-1)^{k} p_{k}(u)=0 .
$$

Similarly to the classical case, these formulae enable us to express power sums via the elementary symmetric polynomials and visa versa. Thus, we can conclude that the power sums also generate the Bethe subalgebra of $\mathbf{Y}(R)$.

In the rational case we respectively define elementary symmetric polynomials and power sums as follows

$$
\begin{gathered}
e_{k}(u)=\operatorname{Tr}_{R(1 \ldots k)}\left(A_{12 \ldots k}^{(k)} L_{\overline{1}}(u) L_{\overline{2}}(u-1) \ldots L_{\bar{k}}(u-k+1)\right), \\
p_{k}(u)=\operatorname{Tr}_{R(1 \ldots k)}\left(L_{\overline{1}}(u-k+1) L_{\overline{2}}(u-k+2) \ldots L_{\bar{k}}(u) R_{k-1} R_{k-2} \ldots R_{1}\right) .
\end{gathered}
$$

They are related by the following analogs of the Newton identities

$$
k e_{k}(u)-p_{1}(u-k+1) e_{k-1}(u)+p_{2}(u-k+2) e_{k-2}(u)+\cdots+(-1)^{k} p_{k}(u)=0 .
$$

Also, in the algebras $\mathbf{Y}(R)$ some analogs of the Cayley-Hamilton identity are valid (see [GS1]) but we do not need them here.

\section{Commutativity of Bethe subalgebra}

In this section we prove that the family of elements $e_{k}(u), 1 \leq k \leq m$, generate a commutative subalgebra in $\mathbf{Y}(R)$. If we expand each $e_{k}(u)$ in a series in the inverse powers of $u$, we get countable set of polynomials in entries of the matrices $L[k]$ commuting with each other.

We begin with some technical results. Let us introduce a notation for specific products (or chains) of current $R$-matrices:

$$
\left[R_{i \rightarrow j}(u)\right]^{( \pm)}= \begin{cases}R_{i}(u) R_{i+1}\left(q^{ \pm 2} u\right) \ldots R_{j}\left(q^{ \pm 2(j-i)} u\right), & \text { if } j \geq i \\ R_{i}(u) R_{i-1}\left(q^{ \pm 2} u\right) \ldots R_{j}\left(q^{ \pm 2(i-j)} u\right), & \text { if } i \geq j .\end{cases}
$$

Analogous notation will be applied to the products of $R^{-1}(u)$. Note, that the above chains of current $R$-matrices are non-singular except for a finite number of isolated values of the parameter $u$ and the inverse chain is given by the formula:

$$
\left\{\left[R_{i \rightarrow j}(u)\right]^{( \pm)}\right\}^{-1}=\left[R_{j \rightarrow i}^{-1}\left(q^{ \pm 2|i-j|} u\right)\right]^{(\mp)} .
$$


Also, note that the middle term in formula (2.1) can be presented as follows

$$
\frac{q^{k}}{k_{q}} I-R_{k}=-R_{k}\left(q^{2 k}\right)
$$

This allows us to get an explicit presentation for the skew-symmetrizer $A^{(k)}$ as a product of chains of $R$-matrices. Namely, we have

Lemma 6 The skew-symmetrizers $A^{(k)}$ defined by (2.1) can be written in the following equivalent forms:

$$
\begin{aligned}
& A_{1 \ldots k}^{(k)}=\frac{(-1)^{\frac{k(k-1)}{2}}}{k_{q} !}\left[R_{1 \rightarrow k-1}\left(q^{2}\right)\right]^{(+)}\left[R_{1 \rightarrow k-2}\left(q^{2}\right)\right]^{(+)} \ldots R_{1}\left(q^{2}\right), \\
& A_{1 \ldots k}^{(k)}=\frac{(-1)^{\frac{k(k-1)}{2}}}{k_{q} !}\left[R_{k-1 \rightarrow 1}\left(q^{2}\right)\right]^{(+)}\left[R_{k-1 \rightarrow 2}\left(q^{2}\right)\right]^{(+)} \ldots R_{k-1}\left(q^{2}\right), \\
& A_{1 \ldots k}^{(k)}=\frac{(-1)^{\frac{k(k-1)}{2}}}{k_{q} !} R_{1}\left(q^{2}\right)\left[R_{2 \rightarrow 1}\left(q^{4}\right)\right]^{(-)} \ldots\left[R_{k-1 \rightarrow 1}\left(q^{2(k-1)}\right)\right]^{(-)}, \\
& A_{1 \ldots k}^{(k)}=\frac{(-1)^{\frac{k(k-1)}{2}}}{k_{q} !} R_{k-1}\left(q^{2}\right)\left[R_{k-2 \rightarrow k-1}\left(q^{4}\right)\right]^{(-)} \ldots\left[R_{k-1 \rightarrow 1}\left(q^{2(k-1)}\right)\right]^{(-)} .
\end{aligned}
$$

Here, we use the standard notation $k_{q} !=1_{q} 2_{q} \ldots k_{q}$.

The lemma can be easily proved by induction in $k$ on the base of (2.1).

Then, with the use of the Yang-Baxter equation (1.2) we can find the following rule for the permutation of $R$-chains:

$$
\left[R_{1 \rightarrow k}(u)\right]^{(+)}\left[R_{1 \rightarrow k-1}\left(q^{2}\right)\right]^{(+)}=\left[R_{2 \rightarrow k}\left(q^{2}\right)\right]^{(+)}\left[R_{1 \rightarrow k-1}\left(q^{2} u\right)\right]^{(+)} R_{k}(u) .
$$

Note, that in the right hand side we have a cyclic permutation of the set of $u$-depending parameters of the corresponding $R$-chain:

$$
\left\{u, q^{2} u, \ldots, q^{2(k-1)} u\right\} \rightarrow\left\{q^{2} u, \ldots, q^{2(k-1)} u, u\right\} .
$$

This observation with formulae of Lemma 6 allows us to prove the following result.

Lemma 7 The $R$-chains commute with the skew-symmetrizers in accordance with the rule:

$$
\begin{aligned}
& {\left[R_{1 \rightarrow k}\left(q^{-2(k-1)} u\right)\right]^{(+)} A_{1 \ldots k}^{(k)}=A_{2 \ldots k+1}^{(k)}\left[R_{1 \rightarrow k}(u)\right]^{(-)},} \\
& {\left[R_{1 \rightarrow k}^{-1}(u)\right]^{(-)} A_{1 \ldots k}^{(k)}=A_{2 \ldots k+1}^{(k)}\left[R_{1 \rightarrow k}^{-1}\left(q^{-2(k-1)} u\right)\right]^{(+)},} \\
& A_{1 \ldots k}^{(k)}\left[R_{k \rightarrow 1}(u)\right]^{(-)}=\left[R_{k \rightarrow 1}\left(q^{-2(k-1)} u\right)\right]^{(+)} A_{2 \ldots k+1}^{(k)}, \\
& A_{1 \ldots k}^{(k)}\left[R_{k \rightarrow 1}^{-1}\left(q^{-2(k-1)} u\right)\right]^{(+)}=\left[R_{k \rightarrow 1}^{-1}(u)\right]^{(-)} A_{2 \ldots k+1}^{(k)} .
\end{aligned}
$$

Let us point out the inverse order of the parameters of the $R$-chains in the left and right hand sides of the above relations, as well as the shift of the spaces in which the skew-symmetrizer acts. 
The last auxiliary result is connected with the chains of generating matrices $L(u)$. First of all, applying (1.8) we get

$$
\begin{aligned}
{\left[R_{k-1 \rightarrow 1}\left(q^{2(k-1)}\right)\right]^{(-)} L_{\overline{1}}(u) L_{\overline{2}}\left(q^{-2} u\right) } & \ldots L_{\overline{k-1}}\left(q^{-2(k-2)} u\right) L_{\bar{k}}\left(q^{-2(k-1)} u\right)= \\
& L_{\overline{1}}\left(q^{-2} u\right) L_{\overline{2}}\left(q^{-4} u\right) \ldots L_{\overline{k-1}}\left(q^{-2(k-1)} u\right) L_{\bar{k}}(u)\left[R_{k-1 \rightarrow 1}\left(q^{2(k-1)}\right)\right]^{(-)} .
\end{aligned}
$$

Then, using Lemma 6 we come to the following result:

$$
A_{1 \ldots k}^{(k)} L_{\overline{1}}(u) L_{\overline{2}}\left(q^{-2} u\right) \ldots L_{\bar{k}}\left(q^{-2(k-1)} u\right)=L_{\overline{1}}\left(q^{-2(k-1)} u\right) L_{\overline{2}}\left(q^{-2(k-2)} u\right) \ldots L_{\bar{k}}(u) A_{1 \ldots k}^{(k)} .
$$

Now, we are ready to prove the main theorem of this paper.

Proposition 8 The elementary symmetric functions (2.7) commute with each other:

$$
e_{k}(u) e_{p}(v)=e_{p}(v) e_{k}(u), \quad \forall k, p \geq 1, \quad \forall u, v .
$$

Proof. In order to simplify formulae we introduce a shorthand notation for a chain of the matrices $L(u)$ with shifted arguments:

$$
\left[L_{\bar{i} \rightarrow \bar{j}}(u)\right]^{( \pm)}=L_{\bar{i}}(u) L_{\overline{i+1}}\left(q^{ \pm 2} u\right) \ldots L_{\bar{j}}\left(q^{ \pm 2(j-i)} u\right) .
$$

Using this notation and the identity (2.8) we can write the left hand side of (3.8) in the form:

$$
e_{k}(u) e_{p}(v)=\operatorname{Tr}_{R(1 \ldots k+p)} A_{1 \ldots k}^{(k)} A_{k+1 \ldots k+p}^{(p)}\left[L_{\overline{1} \rightarrow \bar{k}}(u)\right]^{(-)}\left[L_{\overline{k+1} \rightarrow \overline{k+p}}(v)\right]^{(-)} .
$$

Then, we permute the $u$-depending chain of $L$ matrices with $v$-depending chain with the help of braided Yangian relations (1.8). At the first step of this process we move the utmost right element $L_{\bar{k}}\left(q^{-2(k-1)} u\right)$ through the $v$-chain of the matrices $L$. Relations (1.8) give:

$$
L_{\bar{k}}\left(q^{-2(k-1)} u\right) L_{\overline{k+1}}(v)=R_{k}^{-1}\left(q^{-2(k-1)} u / v\right) L_{\bar{k}}(v) L_{\overline{k+1}}\left(q^{-2(k-1)} u\right) R_{k}\left(q^{-2(k-1)} u / v\right) .
$$

Repeating this procedure we find:

$$
\begin{aligned}
& L_{\bar{k}}\left(q^{-2(k-1)} u\right)\left[L_{\overline{k+1} \rightarrow \overline{k+p}}(v)\right]^{(-)}= \\
& \quad\left[R_{k \rightarrow k+p-1}^{-1}\left(q^{-2(k-1)} x\right)\right]^{(+)}\left[L_{\bar{k} \rightarrow \overline{k+p-1}}(v)\right]^{(-)} L_{\overline{k+p}}\left(q^{-2(k-1)} u\right)\left[R_{k+p-1 \rightarrow k}\left(q^{-2(k-p)} x\right)\right]^{(-)},
\end{aligned}
$$

where $x=u / v$.

Now, we should move the next factor $L_{\overline{k-1}}\left(q^{-2(k-2)} u\right)$ to the right position (it is possible since this factor commutes with the chain of $R^{-1}$-matrices appeared in the above formula) and so on. Thus, we arrive to the expression:

$$
\begin{aligned}
{\left[L_{\overline{1} \rightarrow \bar{k}}(u)\right]^{(-)}\left[L_{\overline{k+1}} \rightarrow \overline{k+p}(v)\right]^{(-)}=} & \\
& \prod_{1 \leq s \leq k}^{\leftarrow}\left[R_{s \rightarrow s+p-1}^{-1}\left(q^{-2(s-1)} x\right)\right]^{(+)}\left[L_{\overline{1} \rightarrow \bar{p}}(v)\right]^{(-)}\left[L_{\overline{p+1} \rightarrow \overline{p+k}}(u)\right]^{(-)} \\
& \prod_{1 \leq r \leq k}^{\rightarrow}\left[R_{r+p-1 \rightarrow r}\left(q^{-2(r-p)} x\right)\right]^{(-)}
\end{aligned}
$$

where we use the following notation for an ordered product of noncommutative factors:

$$
\prod_{1 \leq i \leq k} Q_{i} \equiv Q_{1} Q_{2} \ldots Q_{k}, \quad \prod_{1 \leq i \leq k}^{\leftarrow} Q_{i} \equiv Q_{k} Q_{k-1} \ldots Q_{1}
$$


So, with the use of (3.10) we rewrite (3.9) in the following form

$$
\begin{aligned}
e_{k}(u) e_{p}(v)= & \operatorname{Tr}_{R(1 \ldots k+p)}\left\{A_{1 \ldots k}^{(k)} A_{k+1 \ldots k+p}^{(p)} \prod_{1 \leq s \leq k}^{\leftarrow}\left[R_{s \rightarrow s+p-1}^{-1}\left(q^{-2(s-1)} x\right)\right]^{(+)}\left[L_{\overline{1} \rightarrow \bar{p}}(v)\right]^{(-)}\right. \\
& \left.\times[L \overline{p+1} \rightarrow \overline{p+k}(u)]^{(-)} \prod_{1 \leq r \leq k}^{\rightarrow}\left[R_{r+p-1 \rightarrow r}\left(q^{-2(r-p)} x\right)\right]^{(-)}\right\}
\end{aligned}
$$

Now, we are going to prove that the ordered products of chains of $R$ - and $R^{-1}$-matrices in the above formula cancel each other under the trace operation. Consequently, the right hand side turns into the product $e_{p}(v) e_{k}(u)$.

Note that except for the simplest case $k=p=1$ this cancellation is not straightforward since in (3.11) there is a pair of skew-symmetrizers between two chains of $R$-matrices. First, we permute the skew-symmetrizers with the $R$-matrices which leads to the changes of their ordering. Then, we have to make some identical transformations in order to guarantee that the parameters of the current $R$-matrices and and inverse current $R$-matrices fit each other in a proper way (see (3.2)).

So, we begin with moving the skew-symmetrizers through the chains of $R^{-1}$-matrices with the use of the relations of Lemma 7 . For the skew-symmetrizer $A_{k+1 \ldots k+p}^{(p)}$ this procedure is straightforward (apply the second line of Lemma 7):

$$
A_{k+1 \ldots k+p}^{(p)} \prod_{1 \leq s \leq k}^{\leftarrow}\left[R_{s \rightarrow s+p-1}^{-1}\left(q^{-2(s-1)} x\right)\right]^{(+)}=\prod_{1 \leq s \leq k}^{\leftarrow}\left[R_{s \rightarrow s+p-1}^{-1}\left(q^{-2(s-p)} x\right)\right]^{(-)} A_{1 \ldots p}^{(p)} .
$$

In order to move the second skew-symmetrizer $A_{1 \ldots k}^{(k)}$ we have to reorder the chains of $R$-matrices using the following evident identity:

$$
\prod_{1 \leq s \leq k}^{\leftarrow}\left[R_{s \rightarrow s+p-1}^{-1}\left(q^{-2(s-p)} x\right)\right]^{(-)}=\prod_{1 \leq s \leq p}^{\rightarrow}\left[R_{s+k-1 \rightarrow s}^{-1}\left(q^{-2(s+k-p-1)} x\right)\right]^{(+)}
$$

Now, we can move $A_{1 \ldots k}^{(k)}$ with the help of the last line formula of Lemma 7

$$
A_{1 \ldots k}^{(k)} \prod_{1 \leq s \leq p}^{\rightarrow}\left[R_{s+k-1 \rightarrow s}^{-1}\left(q^{-2(s+k-p-1)} x\right)\right]^{(+)}=\prod_{1 \leq s \leq p}^{\rightarrow}\left[R_{s+k-1 \rightarrow s}^{-1}\left(q^{-2(s-p)} x\right)\right]^{(-)} A_{p+1 \ldots k+p}^{(k)} .
$$

So, we come to the relation:

$$
\begin{aligned}
e_{k}(u) e_{p}(v)= & \operatorname{Tr}_{R(1 \ldots k+p)}\left\{\prod_{1 \leq s \leq k}\left[R_{k+1-s \rightarrow k+p-s}^{-1}\left(q^{-2(s-p)} x\right)\right]^{(-)} A_{1 \ldots p}^{(p)} A_{p+1 \ldots k+p}^{(k)}\left[L_{\overline{1} \rightarrow \bar{p}}(v)\right]^{(-)}\right. \\
& \left.\times\left[L_{\overline{p+1} \rightarrow \overline{p+k}}(u)\right]^{(-)} \prod_{1 \leq r \leq k}^{\rightarrow}\left[R_{r+p-1 \rightarrow r}\left(q^{-2(r-p)} x\right)\right]^{(-)}\right\}
\end{aligned}
$$

where we also reordered the chains of inverse $R$-matrices with the use of identity:

$$
\prod_{1 \leq s \leq p}\left[R_{s+k-1 \rightarrow s}^{-1}\left(q^{-2(s-p)} x\right)\right]^{(-)}=\prod_{1 \leq s \leq k}^{\rightarrow}\left[R_{k+1-s \rightarrow k+p-s}^{-1}\left(q^{-2(s-p)} x\right)\right]^{(-)} .
$$

Next, using the projector property (2.6) we can write

$$
A_{1 \ldots p}^{(p)} A_{p+1 \ldots k+p}^{(k)}=A_{1 \ldots p}^{(p)} A_{p+1 \ldots k+p}^{(k)} A_{1 \ldots p}^{(p)} A_{p+1 \ldots k+p}^{(k)}
$$


and then move the second pair of skew-symmetrizers to the rightmost position. In so doing, we apply (3.7) to pass through the chains of $L$-matrices and Lemma 7 to permute $A^{(k)} A^{(p)}$ with the chains of current $R$-matrices.

At last, with the help of (2.4) we cyclically move the chains of $R$-matrices and the second pair of skew-symmetrizers. Finally, we get the following result:

$$
\begin{aligned}
e_{k}(u) e_{p}(v) & =\operatorname{Tr}_{R(1 \ldots k+p)}\left\{\prod_{1 \leq r \leq k}\left[R_{r+p-1 \rightarrow r}\left(q^{-2(k-r)} x\right)\right]^{(+)} A_{1 \ldots k}^{(k)} A_{k+1 \ldots k+p}^{(p)}\right. \\
& \times \prod_{1 \leq s \leq k}^{\leftarrow}\left[R_{s \rightarrow s+p-1}^{-1}\left(q^{-2(k-p+1-s)} x\right)\right]^{(-)} A_{p+1 \ldots k+p}^{(k)} A_{1 \ldots p}^{(p)} \\
& \left.\times\left[L_{\overline{1} \rightarrow \bar{p}}\left(q^{-2(p-1)} v\right)\right]^{(+)}\left[L_{\overline{p+1} \rightarrow \overline{p+k}}\left(q^{-2(k-1)} u\right)\right]^{(+)}\right\} .
\end{aligned}
$$

Note, that in the above expression the chains of current $R$-matrices are exactly inverse to each other (see (3.2)). The only obstacle for their cancellation is the pair of skew-symmetrizers between them. To remove these skew-symmetrizers, we permute the outer pair $A_{p+1 \ldots k+p}^{(k)} A_{1 \ldots p}^{(p)}$ with the chain of $R^{-1}$-matrices, use the above projector property and then move the pair of skew-symmetrizers back through the chain of inverse $R$-matrices.

Due to such a twice permutation the chains of inverse current $R$-matrices are not changed and all $R$-matrix chains cancel each other. Finally, we get

$$
\begin{aligned}
e_{k}(u) e_{p}(v) & =\operatorname{Tr}_{R(1 \ldots k+p)}\left\{A_{1 \ldots p}^{(p)} A_{p+1 \ldots k+p}^{(k)}\left[L_{\overline{1} \rightarrow \bar{p}}\left(q^{-2(p-1)} v\right)\right]^{(+)}\left[L_{\overline{p+1} \rightarrow \overline{p+k}}\left(q^{-2(k-1)} u\right)\right]^{(+)}\right\} \\
& =\operatorname{Tr}_{R(1 \ldots k+p)}\left\{\left[L_{\overline{1} \rightarrow \bar{p}}(v)\right]^{(-)}\left[L_{\overline{p+1} \rightarrow \overline{p+k}}(u)\right]^{(-)} A_{1 \ldots p}^{(p)} A_{p+1 \ldots k+p}^{(k)}\right\}=e_{p}(v) e_{k}(u) .
\end{aligned}
$$

As was noticed above, on expanding $e_{k}(u)$ and $e_{p}(v)$ in a series in $u^{-1}$ and $v^{-1}$ we can reformulate this commutativity in terms of plynomials in entries of the matrices $L[k]$.

\section{Braided Gaudin models}

In the present section we deal with some versions of the Gaudin model, associated with the braided Yangians considered above. First, we recall the classical version of the rational Gaudin model.

Let $M(k), k=1,2, \ldots, K$, be $m \times m$ matrices subject to the following relations

$$
M(k)_{1} M(l)_{2}=M(l)_{2} M(k)_{1}, \quad k \neq l,
$$

and

$$
M(k)_{1} M(k)_{2}-M(k)_{2} M(k)_{1}=M(k)_{1} P-M(k)_{2} P .
$$

Then the elements

$$
H_{k}=\sum_{l \neq k} \operatorname{Tr} \frac{M(k) M(l)}{u_{k}-u_{l}},
$$

where $u_{k}, k=1,2, \ldots, K$, are pairwise distinct complex numbers, commute with each other.

This claim results from the fact that the elements $H_{k}$ are quadratic Hamiltonians of the rational Gaudin model. The higher Hamiltonians of this model have been constructed by D.Talalaev [T]. His result can be presented as follows.

Let $L(u)=\sum_{k \geq 1} L[k] u^{-k}$ be an $m \times m$ matrix subject to the relation

$$
\left[L_{1}(u), L_{2}(v)\right]=\left[\frac{P}{u-v}, L_{1}(u)+L_{2}(v)\right] .
$$


Then the elements

$$
Q H_{k}(u)=\operatorname{Tr}_{(1 \ldots m)} A_{1 \ldots m}^{(m)}\left(L_{1}(u)-I \frac{d}{d u}\right)\left(L_{2}(u)-I \frac{d}{d u}\right) \ldots\left(L_{k}(u)-I \frac{d}{d u}\right) \triangleright 1
$$

commute with each other:

$$
Q H_{k}(u) Q H_{l}(v)=Q H_{l}(v) Q H_{k}(u) \quad \forall u, v \quad \text { and } \quad \forall k, l .
$$

Here the notation $\triangleright$ means that the above differential operator is applied to the unit. Thus, all terms of the resulting differential operator $\sum_{p}^{k} Q_{p}(u) \partial^{p}$ are cancelled except that which does not contain the derivative.

Now, by using the fact that the matrix $\sum_{i=1}^{K} \frac{M_{i}}{u-u_{i}}$ satisfies the relation (4.4) (a proof of this fact is straightforward) we get a family of Hamiltonians for the rational Gaudin model.

Observe that the system (4.2) means that entries of each matrix $M(k)$ generate a Lie algebra $g l(m)$, while the entries of matrices $M(k)$ and $M(l)$ with $k \neq l$ commute with each other due to (4.1). Thus, the vector space spanned by the entries of all matrices $M(k)$ is endowed with the structure of the Lie algebra $g l(m)^{\oplus K}$.

Now, in the relations (4.1) and (4.2) we replace all indices by their overlined counterparts (see definition (1.5)) assuming $R$ to be an involutive symmetry. Namely, we consider the following relations

$$
M(k)_{\overline{1}} M(l)_{\overline{2}}=M(l)_{\overline{2}} M(k)_{\overline{1}}, \quad k \neq l,
$$

and

$$
M(k)_{\overline{1}} M(k)_{\overline{2}}-M(k)_{\overline{2}} M(k)_{\overline{1}}=M(k)_{\overline{1}} R-M(k)_{\overline{2}} R .
$$

Note that the relations (4.7) define the enveloping algebra of a braided Lie algebra generated by entries of the matrix $M(k)$ as defined in GPS. Our present purpose is to show that the elements

$$
H_{k}=\sum_{l \neq k} \operatorname{Tr}_{R} \frac{M(k) M(l)}{u_{k}-u_{l}}
$$

commute with each other. Emphasize, that in contrast with (4.3) the trace in this formula is braided.

In order to prove the above claim we follow the main lines of the Talalaev's approach but instead of the Drinfeld's Yangian we consider the braided Yangian arising from an involutive symmetry $R$ of bi-rank $(m \mid 0)$.

More precisely, we work with the braided Yangian defined by (1.4) with the following current $R$ matrix:

$$
R(u, v)=R-\frac{h I}{u-v}
$$

which differs from the initial rational current $R$-matrix by the numerical parameter $h$ in the numerator. We denote the corresponding braided Yangian $\mathbf{Y}(R)_{h}$.

Also, we pass to a new generating matrix $\tilde{L}(u)=\left\|\tilde{l}_{i}^{j}(u)\right\|_{1 \leq i, j \leq m}$ connected with the initial one by a linear shift:

$$
L(u)=I+h \tilde{L}(u): \quad l_{i}^{j}(u)=\delta_{i}^{j}+h \tilde{l}_{i}^{j}(u) .
$$

Consequently, we get the following system for the generating matrix $\tilde{L}(u)$ :

$$
\left(R-\frac{h I}{u-v}\right) \tilde{L}(u)_{1} R \tilde{L}(v)_{1}-\tilde{L}(v)_{1} R \tilde{L}(v)_{1}\left(R-\frac{h I}{u-v}\right)=-\left[R, \frac{\tilde{L}_{1}(u)-\tilde{L}_{1}(v)}{u-v}\right] .
$$

In the limit $h \rightarrow 0$ we get an algebra defined by the following system

$$
\left[\tilde{L}_{\overline{1}}(u), \tilde{L}_{\overline{2}}(v)\right]=\left[\frac{R}{u-v}, \tilde{L}_{\overline{1}}(u)+\tilde{L}_{\overline{2}}(v)\right] .
$$


This relation is an analog of the first Sklyanin structure and can be treated in terms of the braided Lie algebras (see GPS]).

In the algebra $\mathbf{Y}(R)_{h}$ we consider the following elements

$$
\hat{e}_{k}(u)=\operatorname{Tr}_{R(12 \ldots m)} A_{12 \ldots m}^{(m)} L_{\overline{1}}(u) L_{\overline{2}}(u-h) \ldots L_{\bar{k}}(u-h(k-1)), \quad k \geq 1 .
$$

For $h=1$ the element $\hat{e}_{k}(u)$ differs from $e_{k}(u)$ defined in (2.11) by a non-trivial multiplier 2 (see GS3] ) and consequently we can conclude that the elements $\hat{e}_{k}(u)$ commute with each other in the algebra $\mathbf{Y}(R)_{h}$.

Next, we expand the elements $\hat{e}_{k}(u)$ in a series in $h$ and introduce the following linear combinations

$$
\tau_{k}(u)=\sum_{p=0}^{k}(-1)^{k-p} \frac{k !}{p !(k-p) !} \hat{e}_{p}(u) .
$$

The corresponding $h$-expansion of $\tau_{k}(u)$ begins with a term proportional to $h^{k}$ (see [T, GS2]). So, we arrive to the following conclusion: the elements

$$
Q H_{k}(u)=\operatorname{Tr}_{R(1 \ldots m)} A_{1 \ldots m}^{(m)}\left(\tilde{L}_{\overline{1}}(u)-I \frac{d}{d u}\right)\left(\tilde{L}_{\overline{2}}(u)-I \frac{d}{d u}\right) \ldots\left(\tilde{L}_{\bar{k}}(u)-I \frac{d}{d u}\right) \triangleright 1
$$

commute with each other in the algebra defined by (4.9).

Also, observe that the matrix

$$
\sum_{k=1}^{K} \frac{M(k)}{u-u_{k}}
$$

satisfies the system (4.9), provided that the matrices $M(k)$ are subject to (4.6) and (4.7). A verification of this property is straightforward.

Consequently, the mutual commutativity of the elements (4.11) remains valid if we replace the matrix $\tilde{L}_{1}(u)$ by $\sum_{k} \frac{M(k)}{u-u_{k}}$ in this formula. As usual, the commutativity of the quadratic Hamiltonians can be deduced from this claim via computing the residues at the points $u_{k}$.

Unfortunately, this method does not lead to new interesting systems when it is applied to braided Yangians related to Hecke symmetries. Indeed, in GS3] a similar linear shift of the generating matrix $L(u)$ was applied in the braided Yangian $\mathbf{Y}(R)$ corresponding to a Hecke symmetry $R$. However, the role of the parameter $h$ in a subsequent expansion was attributed to $\log q$. While $q$ tends to 1 the Hecke symmetry $R$ tends to either the flip $P$ or an involutive symmetry $\tilde{R}$ if $R$ is a deformation of $P$ or $\tilde{R}$ respectively.

Finally, we come to a limit algebra defined by the system

$$
\left[\tilde{L}_{\overline{1}}(u), \tilde{L}_{\overline{2}}(v)\right]=\left[\frac{\tilde{R}}{u-v}, u \tilde{L}_{\overline{1}}(u)+v \tilde{L}_{\overline{2}}(v)\right]
$$

which does not depend on $q$ (in the former case $\tilde{R}=P$ ).

The case $\tilde{R}=P$ was considered in [GS3]. Namely, by using the Talalaev's method, it is easy to construct a Bethe subalgebra in the algebra defined by (4.12). Then, upon applying the morphism

$$
\tilde{L}(u) \mapsto \sum_{k=1}^{K} \frac{M(k) u_{k}}{u-u_{k}},
$$

it is possible to conclude that the elements

$$
H_{k}=\sum_{l \neq k} \operatorname{Tr} \frac{M(k) M(l) u_{l}}{u_{k}-u_{l}}
$$

\footnotetext{
${ }^{2}$ This property is only valid in the braided Yangians of RE type.
} 
commute with each other, provided the matrices $M(k)$ are subject to (4.1) and (4.2). However, this model is not new, since the change of parameters $u_{k} \mapsto u_{k}^{-1}$ reduces this family to that (4.3) above.

In general, i.e. while $\tilde{R} \neq P$, all these considerations remain valid if we assume that the matrices $M(k)$ are subject to (4.6) and (4.7) with $R=\tilde{R}$ and replace the usual trace $\operatorname{Tr}$ in (4.13) by its $\tilde{R}$-counterpart.

\section{References}

[GS1] D.Gurevich, P.Saponov, Braided Yangians, http://lanl.arxiv.org/abs/1612.05929.

[GS2] D.Gurevich, P.Saponov, Generalized Yangians and their Poisson counterparts, Theoretical and Mathematical Physics, 192 (2017), 1097-1111.

[GS3] D.Gurevich, P.Saponov, From Reflection Equation Algebra to Braided Yangians (with P.Saponov), Proceedings of International Conference on Mathematical Physics (Grozny, Russia), to appear.

[GPS] D. Gurevich, P. Pyatov and P. Saponov, Representation theory of (modified) reflection equation algebra of $G L(m \mid n)$ type, St. Petersburg Math. J., vol. 20 (2009) pp. 213-253.

[IOP] A.Isaev, O.Ogievetsky, P.Pyatov, On quantum matrix algebras satisfying the CayleyHamilton-Newton identities, J. Phys. A 32 (1999), no. 9, L115-L121.

[M] A.Molev, Yangians and classical Lie algebras, Mathematical Surveys and Monographs, 143. American Mathematical Society, Providence, RI, 2007.

[T] D.Talalaev, Quantum Gaudin system, Func. Anal. Appl. 40 (2006), no 1, 73-77. 